\title{
Synthesis and characterization of poly(2-ethylhexyl acrylate) prepared via atom transfer radical polymerization, reverse atom transfer radical polymerization and radical polymerization
}

\author{
DHRUBA JYOTI HALOI ${ }^{\mathrm{a}, \mathrm{b}, *}$, BISHNU PRASAD KOIRY $^{\mathrm{b}}$, PRITHWIRAJ MANDAL $^{\mathrm{b}}$ and \\ NIKHIL KUMAR SINGHA ${ }^{\text {b }}$ \\ aZydex Industries, 25-A Gandhi Oil Mill Compound, Gorwa, Vadodara 390 016, India \\ ${ }^{\mathrm{b}}$ Rubber Technology Centre, Indian Institute of Technology Kharagpur, Kharagpur 721 302, India \\ e-mail: dhru_ba2k3@yahoo.co.in
}

MS received 1 December 2012; revised 22 February 2013; accepted 15 April 2013

\begin{abstract}
This investigation reports a comparative study of poly(2-ethylhexyl acrylate) (PEHA) prepared via atom transfer radical polymerization (ATRP), reverse atom transfer radical polymerization (RATRP) and conventional free radical polymerization (FRP). The molecular weights and the molecular weight distributions of the polymers were measured by gel permeation chromatography (GPC) analysis. Structural characterization of the polymers was carried out by ${ }^{1} \mathrm{H}$ NMR and MALDI-TOF-MS analyses. Thermal properties of the polymers were evaluated by differential scanning calorimetry (DSC) and thermogravimetric analysis (TGA). The polymerization results and the thermal properties of PEHAs prepared via ATRP, RATRP and FRP were compared.
\end{abstract}

Keywords. Atom transfer radical polymerization (ATRP); thermal properties; polymer synthesis.

\section{Introduction}

Free radical polymerization (FRP) is the most widely used polymerization method to polymerize almost all vinyl monomers. ${ }^{1}$ The advantages of FRP is that it is compatible with wide range of monomers including speciality functional monomers, more versatile with respect to reaction conditions and is applicable to wide range of polymerization temperature. ${ }^{2}$ The disadvantages of this FRP are (i) uncontrolled molecular weight with broad molecular weight distribution, (ii) very short half-life time of the propagating chain for which it is impossible to prepare block copolymers and (iii) excessive chain transfer and chain termination during FRP often lead to gelation. ${ }^{3,4}$ To overcome these difficulties of FRP, different improvised techniques have been introduced to synthesize polymer with controlled molecular weight, narrow molecular weight distribution with well-defined architecture and topology. ${ }^{5-7}$ These polymerization techniques are known as controlled radical polymerization (CRP) technique. Among the different CRP techniques, the atom transfer radical polymerization (ATRP) is a versatile living polymerization technique, which is more tolerant to the different

*For correspondence impurities and the different functionality present in the reaction system. ${ }^{8}$

Though the ATRP has many advantages over conventional FRP, it has got some disadvantages too. ATRP uses metal halide in its lower oxidation state which is very much air sensitive. The active organic halides, those are used as initiator in ATRP are normally toxic. The main disadvantage of ATRP is the metal contamination in the product. The transition metal catalyst used for the polymerization remains in the product. It introduces complexity in purification to obtain highly pure product. $^{9}$

On the other hand, the reverse atom transfer radical polymerization (RATRP) uses conventional thermal free radical initiators (like azobisisobutyronitrile (AIBN), benzoyl peroxide (BPO) etc.) unlike active alkyl halides in conventional ATRP. ${ }^{8}$ A suitable transition metal halide in its higher oxidation state (e.g. $\mathrm{CuCl}_{2}, \mathrm{CuBr}_{2}$ etc.) which is less sensitive to air is used in RATRP. The RATRP is preferred over ATRP is mainly due to the toxic halide initiators and the easily oxidizable metal catalysts; those are used in ATRP. ${ }^{10}$

2-Ethyl hexylacrylate (EHA) is a very useful monomer for many industrial applications. The homopolymer of EHA (PEHA) has very low glass transition temperature $\left(T_{g}=-65^{\circ} \mathrm{C}\right)$ and has very good film formation property. ${ }^{11}$ The copolymers of PEHA with 
other acrylates, methacrylates, acrylonitrile, maleic acid esters, vinyl acetate, vinyl chloride, etc. find applications in adhesives, chemical intermediates, coatings, leather, plasticizers, plastics and textiles. ${ }^{12,13}$

Heatley et al. ${ }^{14}$ reported the FRP of EHA in cyclohexane. They reported that FRP of EHA led to highly branched PEHA where branching was introduced in the backbone via proton abstraction. Though few reports are available on copolymerization of EHA with other vinyl monomers using ATRP, there was no report solely on ATRP or RATRP of EHA until a publication from our group reporting ATRP of EHA in the presence of nanoclay as additive. ${ }^{15}$ However, there is no report comparing the polymerization results of PEHA prepared via techniques like FRP, ATRP and reverse ATRP.

This investigation reports a comparative study of few polymerization results of PEHA prepared via ATRP, RATRP and FRP in bulk and in solution.

\section{Experimental}

\subsection{Materials}

2-Ethylhexylacrylate (EHA) (98\%, BASF) and methyl methacrylate (MMA) (99\%, Aldrich) were used after removing inhibitor by passing through alumina (basic) packed column. $\mathrm{CuBr}$ (98\%, Aldrich), $\mathrm{CuBr}_{2}$ (97\%, Riedel-deHaën), $\mathrm{CuCl}$ (98\%, Aldrich), 2, 2'-bypyridine (bpy) (98\%, Aldrich) were used as received. Toluene (98\%, Merck) was purified by distilling under reduced pressure. $\mathrm{N}, \mathrm{N}, \mathrm{N}^{\prime}, \mathrm{N}^{\prime \prime}, \mathrm{N}^{\prime \prime}$-pentamethyl diethylenetriamine (PMDETA) (98\%, Aldrich), methyl 2-bromo propionate (MBrP) (99\%, Aldrich), 1phenethyl bromide (PEBr) (99\%, Aldrich), $\mathrm{CDCl}_{3}$ (Aldrich), tetrahydrofuran (THF) (Merck) and acetone (Merck) were used as received. BPO (Merck) was purified by recrystallizing from acetone.

\subsection{Polymerization}

2.2a Homo polymerization of EHA: ATRP of EHA was carried out in bulk. In a typical bulk polymerization, the catalyst $\mathrm{CuCl}(0.0118 \mathrm{~g}, 0.12 \mathrm{mmol})$ and the ligand PMDETA $(0.0311 \mathrm{~g}, 0.18 \mathrm{mmol})$ were added to a Schlenk tube and was sealed properly with a silicone septum. Nitrogen gas was purged through it for several minutes to expel out the oxygen present inside the Schlenk tube. Monomer, EHA (4.43 g, $24 \mathrm{mmol}$ ) was then transferred to the Schlenk tube with continuous purging of nitrogen under continuous stirring. The Schlenk tube was then immersed into an oil bath already heated at $90^{\circ} \mathrm{C}$ and polymerization was started by adding initiator, $\operatorname{MBrP}(0.02 \mathrm{~g}, 0.12 \mathrm{mmol})$. At different time intervals, the aliquots were taken out and conversion was calculated gravimetrically. A part of the aliquot was used to determine its molecular weight via GPC analysis. Its structural characterization and chemical composition were evaluated by ${ }^{1} \mathrm{H}$ NMR analysis. At the end of reaction the polymer sample was dissolved in THF and then passed through the alumina (basic) packed column. The final polymer was dried in a vacuum oven at $50^{\circ} \mathrm{C}$.

ATRP of EHA was also carried out in toluene. In this case, $5 \mathrm{~mL}$ toluene was added to the above reaction mixture used for the bulk polymerization of EHA. RATRP of EHA was also carried out at $90^{\circ} \mathrm{C}$ using the same procedure adopted for the solution ATRP of EHA. In this case used ingredients were: EHA (4.43 g, $24 \mathrm{mmol}), \mathrm{BPO}(0.0290 \mathrm{~g}, 0.12 \mathrm{mmol}), \mathrm{CuBr}_{2}$ $(0.0268 \mathrm{~g}, 0.12 \mathrm{mmol})$, bpy $(0.0374 \mathrm{~g}, 0.24 \mathrm{mmol})$ and toluene $(5 \mathrm{~mL})$. FRP of EHA was carried out in bulk as well as in toluene heating the mixture of EHA $(4.43 \mathrm{~g}, 24 \mathrm{mmol})$ and BPO $(0.0290 \mathrm{~g}, 0.12 \mathrm{mmol})$ at $90^{\circ} \mathrm{C}$ under nitrogen blanketed condition. The polymers were purified by dissolving in THF and passing through the alumina (basic) packed column. The final polymers were dried in a vacuum oven at $50^{\circ} \mathrm{C}$.

\section{2b Chain extension experiment of PEHA: The} PEHA prepared as explained above was used as monofunctional macroinitiator to prepare AB-type di-block copolymer with MMA. In this chain extension experiment, macroinitiator (E-7) $\left(2 \mathrm{~g}, 0.09 \mathrm{mmol}, \mathrm{M}_{\mathrm{n}(\mathrm{GPC})}=\right.$ $21300, \mathrm{PDI}=1.30), \mathrm{CuCl}(0.0092 \mathrm{~g}, 0.09 \mathrm{mmol})$ and PMDETA $(0.0244 \mathrm{~g}, 0.14 \mathrm{mmol})$ in acetone $(1 \mathrm{~mL})$ were added to a Schlenk tube and was sealed with rubber septum. Nitrogen (gas) was passed through the sealed reaction mixture for $10 \mathrm{~min}$. On continuous stirring, MMA ( $4 \mathrm{~mL}, 0.0375 \mathrm{~mol}$ ) was then injected to the reaction mixture. The reaction was started by putting the Schlenk tube in a preheated oil bath at $90^{\circ} \mathrm{C}$. The reaction was stopped after $5 \mathrm{~h}$ and the block copolymer thus prepared via this chain extension reaction was purified using the same technique mentioned in above section. The purified block copolymer was analysed for molecular weight and PDI by GPC analysis.

\subsection{Characterization}

The molecular weights and its distributions of polymers were measured by size exclusion chromatography (SEC) on a VISCOTEK, GPC instrument equipped with VE 1122 solvent delivery system; VE 3580 RI Detector. Tetrahydrofuran (THF) was used as the eluent at a flow rate of $1 \mathrm{~mL} / \mathrm{min}$. Poly(styrene) standards 
were used as calibration standard. ${ }^{1} \mathrm{H}$ NMR spectra of the polymers were recorded on a $200 \mathrm{MHz}$ Brucker NMR spectrometer using $\mathrm{CDCl}_{3}$ as solvent containing a small amount of TMS as internal standard. Thermogravimetric analysis (TGA) was carried out on a TA (TGA Q50 V6.1 Build 181) instrument at a heating rate of $20^{\circ} \mathrm{C} / \mathrm{min}$ under nitrogen atmosphere. Differential scanning calorimetry (DSC) was also carried out on a TA (DSC Q100 V8.1 Build 251) instrument at a heating rate of $20^{\circ} \mathrm{C} / \mathrm{min}$ under nitrogen atmosphere. MALDITOF-MS analysis was carried out using a Perceptive Biosystems Voyager Elite MALDI-TOF mass spectrometer, equipped with a nitrogen laser (wavelength $337 \mathrm{~nm})$. The matrix (2,5-dihydroxybenzoic acid), salt (sodium trifluoroacetate) and polymer solution were premixed in the ratio of 10/1/10.

\section{Results and discussion}

\subsection{Kinetics of polymerization}

The homopolymerization of EHA was carried out in bulk as well as in solution at $90^{\circ} \mathrm{C}$ via ATRP. Figure 1 shows the kinetic plot of $\ln (1-\mathrm{x})^{-1}$ vs polymerization time for ATRP of EHA in bulk. This linear kinetic plot indicates the controlled nature of the polymerization indicating the concentration of active species remains constant throughout the polymerization. Figure 2 shows the plot of $\mathrm{M}_{\mathrm{n}(\mathrm{GPC})}$ and PDI versus conversion of ATRP of EHA in bulk. The raw GPC data of the kinetic plots are shown in table $\mathrm{S} 1$ in the supplementary section. The linear increase in molecular weight with monomer conversion confirmed the controlled nature of the polymerization. The ATRP of EHA

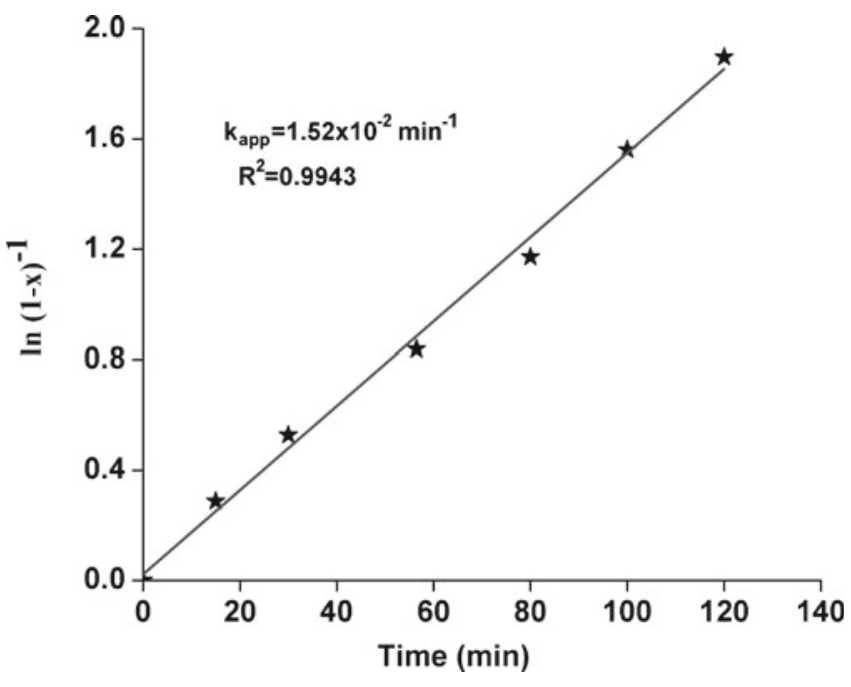

Figure 1. Kinetic plot of ATRP of EHA (E-1) in bulk at $90^{\circ} \mathrm{C}$.

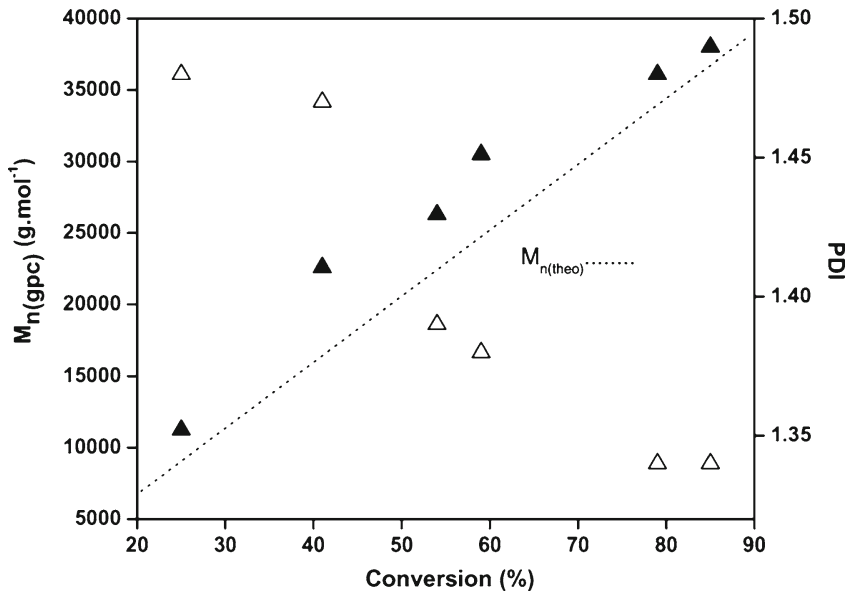

Figure 2. Plot of $M_{n(G P C)}$ and PDI versus conversion of EHA in bulk ATRP (E-1).

was carried out using $\mathrm{CuCl} / \mathrm{PMDETA}$ as catalyst system and $\mathrm{MBrP}$ as initiator. Acrylates having long chain alkyl pendant group is difficult to polymerize via ATRP in a controlled manner. Many research groups reported that addition of small amount of polar solvents, like acetone, diethyl carbonate, water, DMSO, DMF, etc. helps to control the ATRP of acrylates having long chain alkyl pendant group. ${ }^{16}$ In our case, acetone was used as an additive at $20 \% \mathrm{~V} / \mathrm{V}$ with respect to the monomer amount. Table 1 shows the polymerization results of ATRP of EHA in bulk. The rate of the polymerization was slow when $\mathrm{CuCl} / \mathrm{bpy}$ was used as catalyst system with small amount of acetone. It led to low conversion and exhibited broad molecular weight distribution. The rate of ATRP of EHA was enhanced when $\mathrm{CuCl} / \mathrm{bpy}$ catalyst system was replaced by $\mathrm{CuCl} / \mathrm{PMDETA}$ catalyst system. $\mathrm{CuCl} / \mathrm{PMDETA}$ along with acetone as additive exhibited very good control over molecular weight with narrow molecular weight distribution. The polymerization was found to be much controlled when $\mathrm{CuBr} / \mathrm{PMDETA}$ was used as catalyst system along with acetone as additive and $\mathrm{PEBr}$ as initiator. In this case, the polymerization followed a very controlled but slow polymerization pathway.

The ATRP of EHA was also carried out in toluene at $90^{\circ} \mathrm{C}$. Table 2 summarizes the polymerization results of ATRP of EHA in toluene. The polymerization result obtained for $\mathrm{CuCl} / \mathrm{bpy}$ catalyst system along with $\mathrm{MBrP}$ as initiator was improved when $\mathrm{CuBr} / \mathrm{PMDETA}$ catalyst system was used in place of $\mathrm{CuCl} / \mathrm{bpy}$. In all the cases, a small amount of acetone as additive ( $20 \% \mathrm{~V} / \mathrm{V}$ with respect to monomer) further improved the polymerization results.

The reverse ATRP (RATRP) of EHA was also carried out at $90^{\circ} \mathrm{C}$ in toluene using $\mathrm{CuBr}_{2}$ as catalyst in combination with bpy as ligand and benzoyl peroxide 
Table 1. ATRP of 2-ethylhexyl acrylate (EHA) in bulk at $90^{\circ} \mathrm{C}$.

\begin{tabular}{|c|c|c|c|c|c|c|}
\hline Expt. code No & Polymerization system & Time $(\mathrm{h})$ & Conv $(\%)$ & $\mathrm{M}_{\mathrm{n}(\mathrm{GPC})} \mathrm{g} \cdot \mathrm{mol}^{-1}$ & $\mathrm{M}_{\mathrm{n}(\mathrm{Theo})}^{\#} \mathrm{~g} \cdot \mathrm{mol}^{-1}$ & PDI \\
\hline E-1 & $\begin{array}{c}{[\mathrm{EHA}] /[\mathrm{CuCl}] /[\mathrm{PMDETA}] /[\mathrm{MBrP}]} \\
\text { Acetone }(1 \mathrm{~mL}), 200 / 1 / 1.5 / 1\end{array}$ & 4 & 85 & 38000 & 31440 & 1.34 \\
\hline E-2 & $\begin{array}{c}{[\mathrm{EHA}] /[\mathrm{CuCl}] /[\mathrm{bpy}] /[\mathrm{MBrP}]} \\
\text { AcetoneAcetone }(1 \mathrm{~mL}), 200 / 1 / 2 / 1\end{array}$ & 5 & 69 & 37000 & 25550 & 1.48 \\
\hline E-3 & $\begin{array}{c}{[\mathrm{EHA}] /[\mathrm{CuBr}] /[\mathrm{PMDETA}] /[\mathrm{PEBr}]} \\
\text { Acetone }(1 \mathrm{~mL}), 100 / 1 / 2 / 1\end{array}$ & 4 & 41 & 2800 & 7720 & 1.13 \\
\hline
\end{tabular}

${ }^{\#} \mathrm{M}_{\mathrm{n}(\mathrm{Theo})}=\left[\mathrm{DP} * \mathrm{M}_{(\mathrm{EHA})}\right] *$ Conv $(\%)+\mathrm{M}_{(\text {Initiator })} ; \mathrm{DP}=$ Degree of polymerization; $\mathrm{M}_{(\mathrm{EHA})}=$ Molecular weight of EHA; Conv $(\%)=$ Percentage of conversion; $\mathbf{M}_{(\text {Initiator })}=$ Molecular weight of the initiator

Table 2. Polymerization of 2-ethylhexyl acrylate (EHA) in toluene at $90^{\circ} \mathrm{C}$ via ATRP and RATRP.

\begin{tabular}{|c|c|c|c|c|c|c|}
\hline Expt. code No & Polymerization system & Time (h) & Conv $(\%)$ & $\mathrm{M}_{\mathrm{n}(\mathrm{Gpc})} \mathrm{g} \cdot \mathrm{mol}^{-1}$ & $\mathrm{M}_{\mathrm{n}(\text { Theo) }}^{\$} \mathrm{~g} \cdot \mathrm{mol}^{-1}$ & PDI \\
\hline E-4 & $\begin{array}{c}{[\mathrm{EHA}] /[\mathrm{CuCl}] /[\mathrm{bpy}] /[\mathrm{MBrP}]} \\
200 / 1 / 2 / 1\end{array}$ & 10 & 84 & 17200 & 31070 & 1.44 \\
\hline E-5 & $\begin{array}{c}{[\mathrm{EHA}] /[\mathrm{CuBr}] /[\mathrm{PMDETA}] /[\mathrm{MBrP}]} \\
200 / 1 / 1.5 / 1\end{array}$ & 4 & 40 & 6700 & 14880 & 1.39 \\
\hline E-6 & $\begin{array}{c}{[\mathrm{EHA}] /[\mathrm{CuCl}] /[\mathrm{bpy}] /[\mathrm{MBrP}]} \\
\text { Acetone }(1 \mathrm{~mL}), 200 / 1 / 2 / 1\end{array}$ & 8 & 84 & 22100 & 31070 & 1.38 \\
\hline E-7 & $\begin{array}{c}{[\mathrm{EHA}] /[\mathrm{CuCl}] /[\mathrm{PMDETA}] /[\mathrm{MBrP}]} \\
\text { Acetone }(1 \mathrm{~mL}), 200 / 1 / 1.5 / 1\end{array}$ & 6 & 79 & 21300 & 29230 & 1.30 \\
\hline E-8 & $\begin{array}{c}{[\mathrm{EHA}] /\left[\mathrm{CuBr}_{2}\right] /[\mathrm{bpy}] /[\mathrm{BPO}]} \\
200 / 1 / 2 / 1\end{array}$ & 10 & 42 & 21200 & 15530 & 1.50 \\
\hline
\end{tabular}

${ }^{\$} \mathrm{M}_{\mathrm{n}(\mathrm{Theo})}=\left[\mathrm{DP} * \mathrm{M}_{(\mathrm{EHA})}\right] * \mathrm{Conv}(\%)+\mathrm{M}_{(\mathrm{Ini})} ; \mathrm{DP}=$ Degree of polymerization; $\mathrm{M}_{(\mathrm{EHA})}=$ Molecular weight of EHA; Conv $(\%)=$ Percentage of conversion; $\mathrm{M}_{(\mathrm{Ini})}=$ Molecular weight of the portion of the initiator attached to the PEHA chains

Table 3. FRP of 2-ethylhexyl acrylate (EHA) at $90^{\circ} \mathrm{C}$.

\begin{tabular}{|c|c|c|c|c|c|c|}
\hline $\begin{array}{l}\text { Expt. } \\
\text { code No }\end{array}$ & $\begin{array}{l}\text { Polymerization system } \\
{[\text { [EHA]/[BPO] }}\end{array}$ & Time (min) & Conv, $(\%)$ & $\mathrm{M}_{\mathrm{n}(\mathrm{GPC})} \mathrm{g} \cdot \mathrm{mol}^{-1}$ & $\mathrm{M}_{\mathrm{n}(\text { Theo })}^{*} \mathrm{~g} \cdot \mathrm{mol}^{-1}$ & PDI \\
\hline E-9 & 200/1, (Bulk) & 15 & 99 & 13600 & 36510 & 8.4 \\
\hline E-10 & 200/1, ( Solution) & 15 & 89 & 23700 & 32830 & 3.0 \\
\hline
\end{tabular}

${ }^{*} \mathrm{M}_{\mathrm{n}(\mathrm{Theo})}=\left[\mathrm{DP} * \mathrm{M}_{(\mathrm{EHA})}\right] *$ Conv $(\%)+\mathrm{M}_{(\mathrm{Ini})} ; \mathrm{DP}=$ Degree of polymerization; $\mathrm{M}_{(\mathrm{EHA})}=$ Molecular weight of EHA; Conv $(\%)=$ Percentage of conversion; $\mathbf{M}_{(\mathrm{Ini})}=$ Molecular weight of the portion of the initiator attached to the PEHA chains

(BPO) as thermal initiator. Table 2 provides the result of RATRP of EHA. The rate of RATRP was found to be much slower than other polymerizations as shown in tables 1 and 2. The poor control over molecular weight and broad polydispersity index for this polymerization may be due to the presence of some side reactions. These side reactions are assumed to be triggered by the abstraction of the tertiary protons present in the pendant part of EHA by some excess free radicals present in the polymerization system. The EHA used for the polymerizations was made inhibitor free by passing through alumina (basic) packed column. Thus the obtained EHA was not in its highest purity level as it was not distilled to remove the other impurities present. But the ATRP of this inhibitor free EHA showed good control over molecular weight and narrow molecular weight distribution when $\mathrm{CuCl} / \mathrm{PMDETA}$ catalyst system was used along with acetone as additive. $\mathrm{CuBr}$ /PMDETA catalyst system with PEBr as ATRP initiator had shown much better control in molecular weight as compared to the ATRP of EHA when MBrP was used as initiator. But the conversion was found to be very low in this case. This is expected as the styryl radicals are less reactive than acrylate radicals.

The conventional FRP of EHA was also carried out at $90^{\circ} \mathrm{C}$ in toluene as well as in bulk. Table 3 shows the FRP results of EHA in bulk and in solution. Benzoyl peroxide (BPO) was used as thermal initiator in 
both cases. The polymerizations were very fast and very high conversion was achieved within 15 min of reaction time. Polydispersity index was also found to be very high for both cases. This is mainly due to the presence of excessive chain termination. The PEHA prepared by bulk polymerization has very broad PDI. This is due to the chain transfer to monomer. The monomers having longer alkyl chain is prone to this kind of chain transfer as reported by Heatley et al. ${ }^{14}$ During polymerization in toluene this kind of chain termination is minimized. It leads to lower PDI but bit larger molecular weight (table 3).

\subsection{End group analysis}

Figure 3 shows the ${ }^{1} \mathrm{H}$ NMR spectra of PEHA prepared via ATRP and RATRP. The designation of the different protons of PEHA is shown in figure 3. The resonance at $\delta=1.27 \mathrm{ppm}$ (designated as ' 5 ' in figure 3$)$ is due to the methylene $\left(>\mathrm{CH}_{2}\right)$ protons and the peaks at $\delta=0.90$ to $0.83 \mathrm{ppm}$ are due to the methyl protons (designated as ' 6 ') of the PEHA. The resonance at $\delta=3.93 \mathrm{ppm}$ is due to the $-\mathrm{OCH}_{2}-$ protons (designated as ' 1 ') of PEHA. Resonances at $\delta=2.00$ to $1.61 \mathrm{ppm}$ are due to the two types of protons (designated as ' $3+4$ ') present in the PEHA. The resonance at $\delta=2.30 \mathrm{ppm}$ is due to the methine proton present in the backbone of the PEHA (designated as ' 2 '). A small resonance at $\delta=4.40 \mathrm{ppm}$ represents the $>\mathrm{CH}-X$ proton. This ${ }^{1} \mathrm{H}$ NMR study concluded that the PEHA prepared via ATRP and RATRP were structurally identical. The presence of a small resonance around $\delta=$ $4.45 \mathrm{ppm}$ in both ${ }^{1} \mathrm{H}$ NMR spectra indicated that the ATRP and RATRP of EHA was effectively controlled leading to active halogen group at the end.

The presence of living halogen end group in PEHA macromolecular chain was further confirmed by chain extension experiment. In this case the isolated and purified PEHA was used as a macroinitiator for the synthesis of diblock copolymer PEHA- $b$-PMMA. This macroinitiator (PEHA, $\mathrm{M}_{\mathrm{n}(\mathrm{GPC})}=21300, \mathrm{PDI}=1.30$ ) (E-7) initiated the polymerization of methyl methacrylate (MMA) at $90^{\circ} \mathrm{C}$ using $\mathrm{CuCl}$ as catalyst in combination with PMDETA as ligand and acetone as additive. Figure 4 shows the GPC traces of the diblock copolymer. The complete shift of the peak towards the higher molecular weight region after block copolymerization
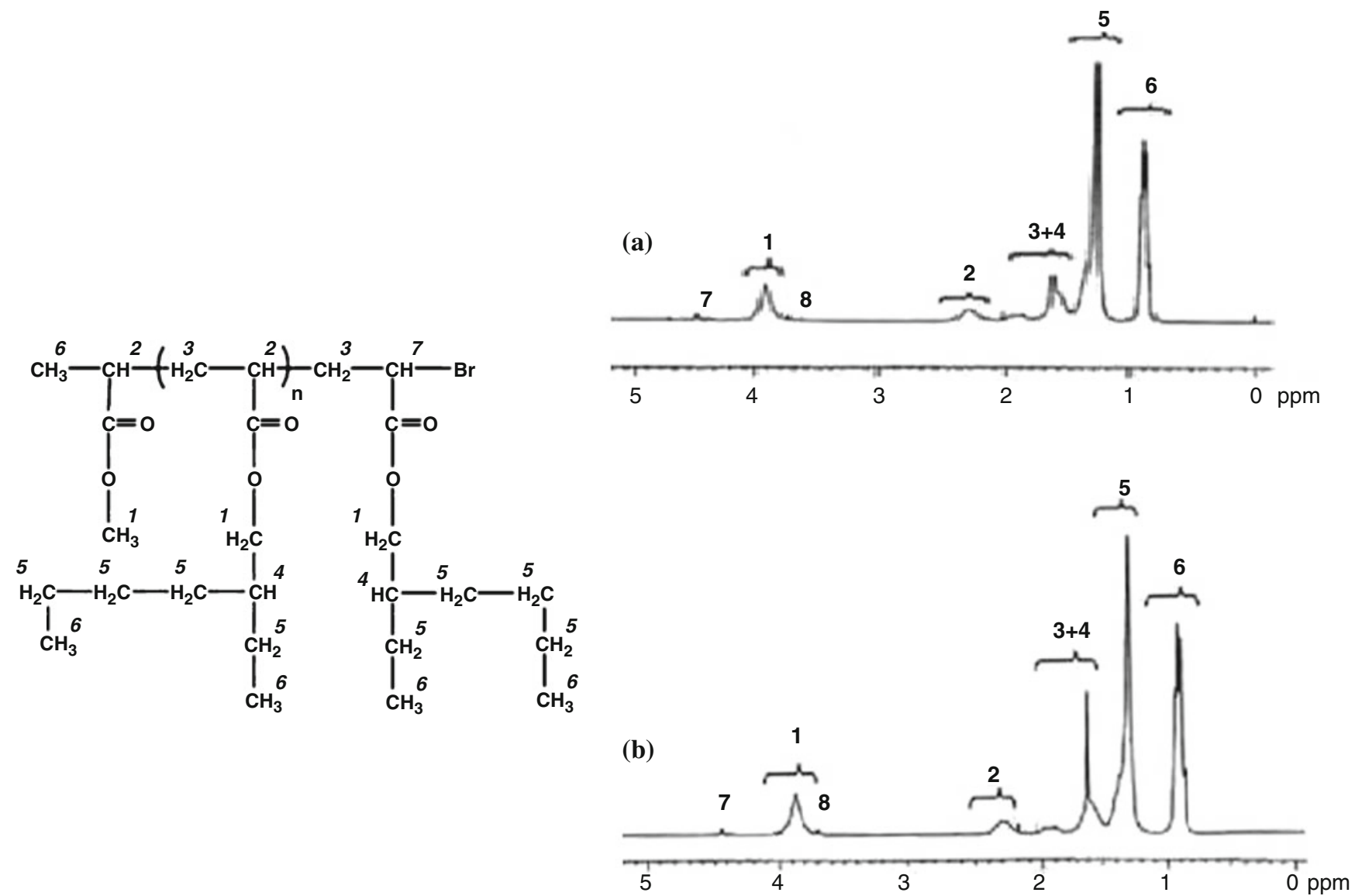

Figure 3. ${ }^{1} \mathrm{H}$ NMR spectra of PEHA (a) solution RATRP and (b) solution ATRP. 


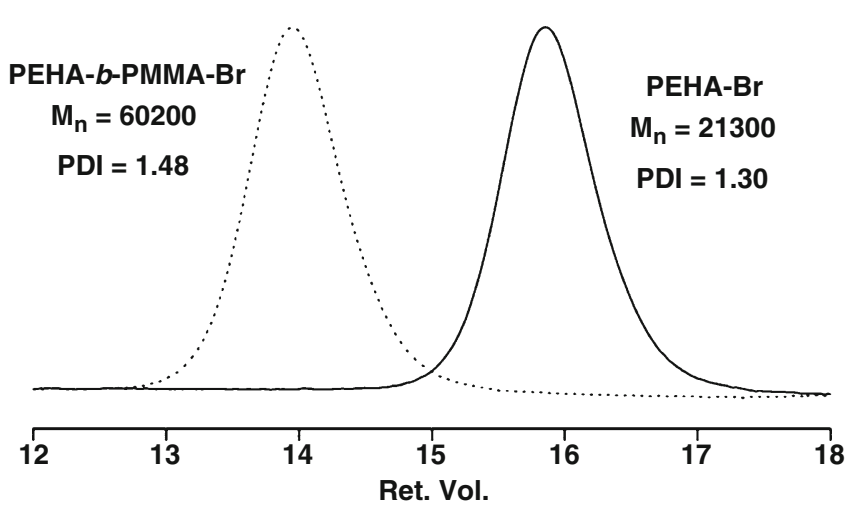

Figure 4. GPC traces of PEHA (E-7) and chain extended PEHA (PEHA- $b$-PMMA).

indicated the presence of active halogen end group in the PEHA macromolecular chains. This indicated that the ATRP of EHA was well controlled and the resultant PEHA had well defined living end group.

Figure 5 shows a selected part of MALDI-TOF mass spectrum of PEHA prepared via solution ATRP. The spectrum shows a constant difference of 184 mass unit in two consecutive major and minor peaks according to their occurrence in the spectrum. 184 is the molar mass of an EHA repeating unit. For example, the peak at 2052 can be assigned as the 'halogenated unit', which has styrene part of $\mathrm{PEBr}$ initiator in one end and $-\mathrm{Br}$ group is in other end. The number of repeating units of EHA in between these two end groups is 10 . The same unit after dehalogenation gives a minor peak at 1972 . The mass difference of these two consecutive major and minor peaks (2052-1972) is 80. This is the mass of one bromine atom. Thus the MALDI-TOF mass analysis of PEHA also confirms the presence of living group at the

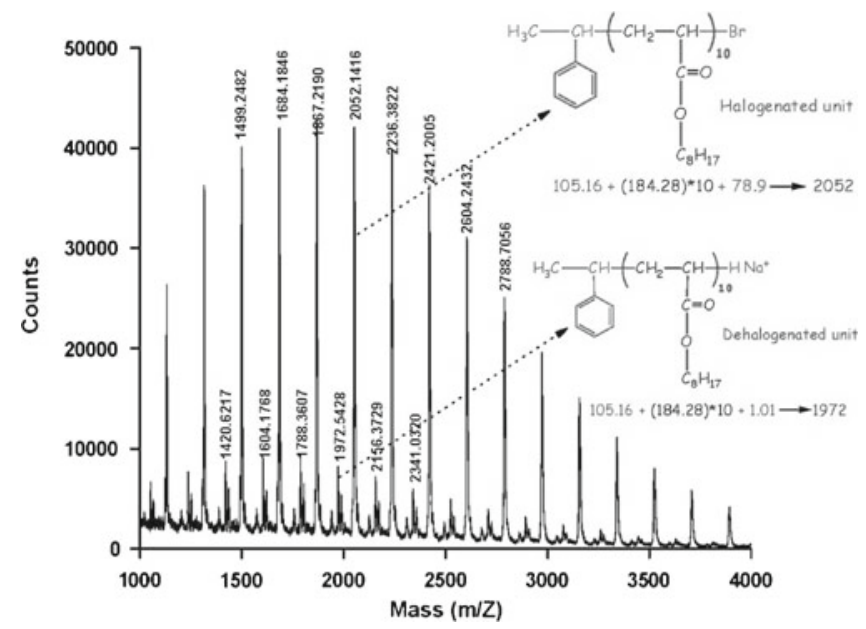

Figure 5. MALDI-TOF mass spectrum of PEHA.

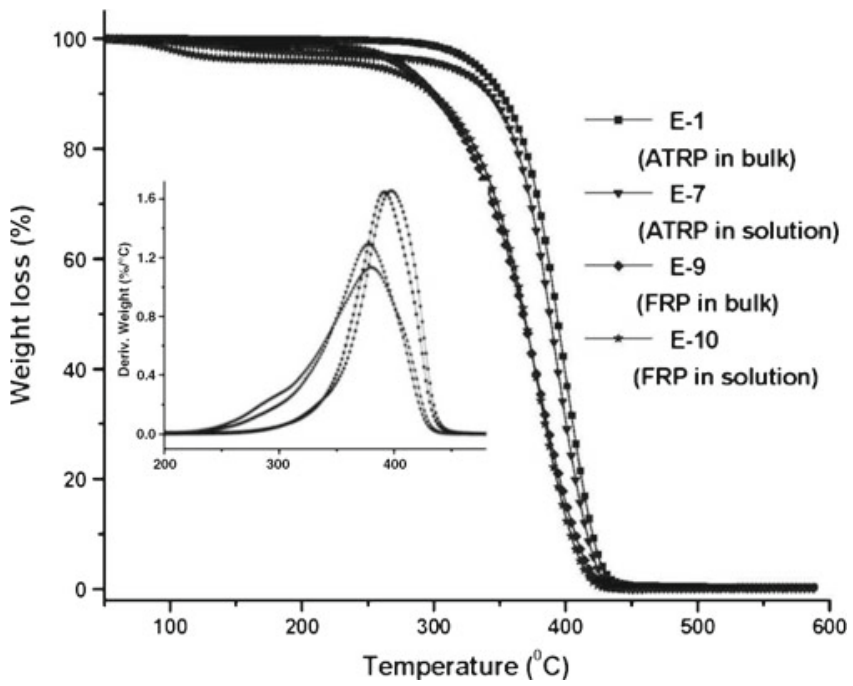

Figure 6. TGA and DTG thermograms of PEHA.

end of PEHA chains and hence the living nature of the polymerization.

\subsection{Thermal property}

3.3a Thermogravimetric analysis: Thermogravimetric analysis (TGA) was carried out to study the thermal stability of the PEHAs prepared via different methods. Figure 6 shows the comparative TGA thermograms of PEHA prepared via ATRP and FRP. This shows the PEHA prepared by FRP has bimodal and broad DTG curves indicating more than one stages of decomposition. The polymers prepared by FRP have vinylic end group (due to termination by disproportion $)^{17}$ and have uncontrolled mode of addition, i.e., head-to-head, tail-to-tail linkage in addition to headto-tail. So the polyacrylates prepared via FRP show lower thermal stability and multi-step decomposition pattern in the TGA analysis. The thermal properties of the PEHA prepared via different techniques are summarized in table 4 . The $T_{\max }$ for the PEHA prepared via ATRP was found to be higher than the PEHA prepared via FRP. Because in ATRP, different side reactions like termination, uncontrolled mode of addition and formation of vinylic end group are absent.

Table 4. Thermal properties of the PEHAs.

\begin{tabular}{lcc}
\hline Code No. & $T_{\max }\left({ }^{\circ} \mathrm{C}\right)$ & $T_{\mathrm{g}}\left({ }^{\circ} \mathrm{C}\right)$ \\
\hline E-1 (ATRP in bulk) & 397 & -66 \\
E-7 (ATRP in solution) & 391 & -69 \\
E-9 (FRP in bulk) & 380 & -66 \\
E-10 (FRP in solution) & 378 & -72 \\
\hline
\end{tabular}


3.3b Glass transition temperature $\left(T_{g}\right)$ : Figure $\mathrm{S} 1$ (in supplementary section) shows the DSC plots of PEHA prepared via different techniques. The glass transition temperature $\left(T_{g}\right)$ is a function of number of free segments present per unit volume of a polymer. It is expected to observe a lower glass transition temperature for PEHA prepared via FRP than PEHA prepared via ATRP as FRP introduces more branching in the resultant polymer. However, that trend was not distinct for the PEHA prepared via different techniques. The $T_{g}$ of PEHA prepared via solution and bulk ATRP was found to be $-69^{\circ} \mathrm{C}$ and $-66^{\circ} \mathrm{C}$, respectively and the $T_{g}$ of PEHA prepared via solution and bulk FRP was found to be $-72^{\circ} \mathrm{C}$ and $-66^{\circ} \mathrm{C}$, respectively. The $T_{g}$ of PEHA prepared by solution polymerization was lower than the $T_{g}$ of PEHA prepared by bulk polymerization. It may be due to the presence of highly branched PEHA chains of different chain length present in the PEHA prepared via solution polymerization. It was resulted from the excessive chain transfer type of reactions with the solvent occurred during polymerization.

\section{Conclusions}

ATRP of 2-ethylhexyl acrylate was successfully carried out at $90^{\circ} \mathrm{C}$ in bulk as well as in toluene via ATRP and RATRP. Good control over molecular weight and narrow molecular weight distribution was achieved when $\mathrm{CuCl} / \mathrm{PMDETA}$ was used as catalyst system along with a small amount of acetone as polar solvent. For the same reaction condition and same catalyst system, ATRP of EHA in bulk produced better polymerization result than ATRP of EHA in toluene. ${ }^{1} \mathrm{H}$ NMR spectra, chain extension experiment and MALDI-TOF analyses indicated that the PEHA prepared via ATRP had well defined active bromide end group. Conventional FRP of EHA in bulk as well as in solution led to polymers with uncontrolled molecular weights and very broad polydispersity. The PEHA prepared via ATRP was thermally more stable than PEHA prepared via FRP.

\section{Supplementary information}

The electronic supplementary information (figure S1 and table S1) can be seen in www.ias.ac.in/chemsci.

\section{Acknowledgement}

Authors are thankful to the Indian Institute of Technology, Kharagpur, India for the financial support.

\section{References}

1. Moad G and Solomon D H 1995 The chemistry of free radical polymerization, 1st edition, Oxford: Elsevier Science

2. Matyjaszewski K and Davis T P 2002 Handbook of radical polymerization, 1st edition, Hoboken: John Wiley

3. Matyjaszewski K 1998, Controlled radical polymerization. ACS Symposium Series 685, American Chemical Society, Washington DC, USA

4. Kamigaito M, Ando T and Sawamoto M 2001 Chem. Rev. 1013689

5. Min K and Matyjaszewski K 2009 Cent. Eur. J. Chem. 7 657

6. Bas S and Soucek M D 2012 Polym. J. 441087

7. Miura Y, Satoh K, Kamigaito M and Okamoto Y 2006 Polym. J. 38930

8. Braunecker W A and Matyjaszewski K 2007 Prog. Polym. Sci. 3293

9. Min K, Gao H and Matyjaszewski K 2005 J. Am. Chem. Soc. 1273825

10. Pan K, Jiang L, Zhang J and Dan Y 2007 J. Appl. Polym. Sci. 105521

11. Haloi D J, Roy S and Singha N K 2009 J. Polym. Sci., Part A: Polym. Chem. 476526

12. Bicak, N. Karagoz, B. and Emre, D. 2006, J. Polym. Sci., Part A: Polym. Chem. 441900

13. Haloi D J, Ata S, Singha N K, Jehnichen D and Voit B 2012 ACS Appl. Mater. Interfaces. 44200

14. Heatley F, Lovell P A and Yamashita T 2001 Macromolecules $\mathbf{3 4} 7636$

15. Haloi D J and Singha N K 2011 J. Polym. Sci., Part A: Polym. Chem. 491564

16. Datta H and Singha N K 2008 J. Polym. Sci., Part A: Polym. Chem. 463499

17. Kashiwagi T, Inaba A, Brown J E, Hatada K, Kitayama T and Masuda E 1986 Macromolecules 192160 